\title{
Solutions to a multi-phase model of sea-ice growth
}

\author{
Yangxin Tang ${ }^{1}$, Lin Zheng ${ }^{1}$, and Liping Luan ${ }^{2}$ \\ ${ }^{1}$ Anhui University of Finance and Economics \\ ${ }^{2}$ Shanghai University
}

March 27, 2021

\begin{abstract}
The multi-phase systems has found its applications in many fields. We shall apply this approach to investigate the multi-phase dynamics of sea-ice growth. In this paper, the weak solutions existence and uniqueness of parabolic differential equations are proved. Then large-time behavior of solutions are studied, also the existence of global attractor is proved. The key tool in this article is the energy method. Our existence proof is only in one dimension.
\end{abstract}

\section{Hosted file}

file-tang-2020.pdf available at https://authorea.com/users/404334/articles/515549-solutionsto-a-multi-phase-model-of-sea-ice-growth 